\title{
ON THE FREE SPECTRUM OF THE VARIETY GENERATED BY THE COMBINATORIAL COMPLETELY 0-SIMPLE SEMIGROUPS
}

\author{
KAMILLA KÁTAI-URBÁN \\ Bolyai Institute, University of Szeged, Aradi vértanúk tere 1, H-6720 Szeged, Hungary \\ e-mail:katai@math.u-szeged.hu \\ and CSABA SZABÓ \\ Eötvös Loránd University, Department of Algebra and Number Theory, 1117 Budapest, \\ Pázmány Péter sétány $1 / \mathrm{c}$, Hungary \\ e-mail:csaba@cs.elte.hu
}

(Received 10 March, 2006; revised 16 September, 2006; accepted 15 March, 2006)

\begin{abstract}
We give an asymptotic bound for the size of the $n$-generated relatively free semigroup in the variety generated by all combinatorial strictly 0 -simple semigroups.
\end{abstract}

2000 Mathematics Subject Classification. 20M05, 08B20.

1. Introduction. For finite algebras there are strong connections between the structural properties of the algebra and the free spectra. If $\mathbf{G}$ is a finite group, then the size of the $n$-generated relatively free group in the variety generated by $\mathbf{G}$ is polynomial in $n$ if and only if $\mathbf{G}$ is nilpotent and at least doubly-exponential if $\mathbf{G}$ is not nilpotent ([3] and [8]).

Let $\mathbf{A}$ be a $k$-element finite algebra and let $\mathcal{V}$ denote the variety generated by $\mathbf{A}$. It is known that the size of the free algebra in $\mathcal{V}$ freely generated by $n$ free elements $\left(\left|\mathbf{F}_{\mathcal{V}}(n)\right|\right)$ is less than $k^{k^{n}}$. If $k \geq 2$, then this number is greater than $n$. The free spectrum of a variety $\mathcal{V}$ is the sequence of cardinalities $\left|\mathbf{F}_{\mathcal{V}}(n)\right|, n=0,1,2, \ldots$ For example the free spectrum of Boolean algebras is $\left|\mathbf{F}_{\mathcal{V}}(n)\right|=2^{2^{n}}$. The first important question about free spectra is the following: within the above bounds what are the possible numbers?

Another theorem on free spectra is Theorem 12.3 in [4]. If $\mathcal{V}$ is a nontrivial locally finite congruence distributive variety, then for every $c$ such that $0<c<1$, and for every large $n$, the free spectrum of $\mathcal{V}$ is bounded below by $2^{2^{c n}}$. There are so called gap theorems for the free spectra, as well. At the lower end, for example, there is Theorem 12.2 in [4] which states the following. Let $\mathcal{V}$ be a variety generated by a $k$-element algebra. Then either $\left|\mathbf{F}_{\mathcal{V}}(n)\right| \leq c n^{k}$ for some finite $c$, or else $\left|\mathbf{F}_{\mathcal{V}}(n)\right| \geq 2^{n-k}$ for all $n$.

For simple algebras there is a characterization of possible free spectra using tame congruence theory. The tame congruence types are denoted by 1, 2, 3, 4 or 5 and called unary, affine, Boolean, lattice or semilattice type, respectively. The next result of Joel Berman is taken from [1]: 
THEOREM 1.1. For each $k \geq 2$ there exist positive constants $d_{1}, \ldots, d_{5}$ and $c_{1}, c_{2}, c_{4}$ such that if $\mathbf{A}$ is a k-element simple algebra and $\mathcal{V}$ is generated by $\mathbf{A}$, then for every sufficiently large $n$,

(1) if $\operatorname{typ}(\mathbf{A})=\mathbf{1}$, then $d_{1} n \leq\left|\mathbf{F}_{\mathcal{V}}(n)\right| \leq c_{1} n^{\log _{2} k}$;

(2) if $\operatorname{typ}(\mathbf{A})=\mathbf{2}$, then $d_{2} k^{n} \leq\left|\mathbf{F}_{\mathcal{V}}(n)\right| \leq c_{2} k^{(k-1) n}$;

(3) if $\operatorname{typ}(\mathbf{A})=\mathbf{3}$, then $k^{d_{3} k^{n}} \leq\left|\mathbf{F}_{\mathcal{V}}(n)\right| \leq k^{k^{n}}$;

(4) if $\operatorname{typ}(\mathbf{A})=\mathbf{4}$, then $k^{d_{4} k^{n} / \sqrt{n}} \leq\left|\mathbf{F}_{\mathcal{V}}(n)\right| \leq k^{c_{4} k^{n} / \sqrt{n}}$;

(5) if $\operatorname{typ}(\mathbf{A})=\mathbf{5}$, then $d_{5} k^{n} \leq\left|\mathbf{F}_{\mathcal{V}}(n)\right| \leq k^{\sigma(n)}$ for

$$
\sigma(n)=\frac{n k}{n-k(k-1)^{3}}\left(\begin{array}{c}
n \\
(k-1)^{3}
\end{array}\right)(k-1)^{n-(k-1)^{3}} .
$$

As we see, for type 5 there is a huge gap between the two bounds. A very interesting class of type 5 algebras is the class of completely 0 -simple semigroups. Completely 0 simple semigroups are one of the basic building blocks for semigroups like simple groups for groups. A semigroup is called combinatorial if it contains no nontrivial proper subgroups. The first natural step in investigating free spectra of semigroups is to find the free spectrum of the variety generated by the combinatorial completely 0 -simple semigroups. In this paper we prove that the free spectrum of this variety is asymptotically $2^{n^{2}+2 \log n}$.

Every completely 0-simple semigroup is isomorphic to a so called Rees matrix semigroup. A Rees matrix semigroup over the group $G$ adjoined with a $0, G^{0}(=G \cup\{0\})$, is constructed in the following way: Let $I, \Lambda$ be non-empty sets, and let $P=\left(p_{\lambda i}\right)$ be a $\Lambda \times I$ matrix over $G^{0}$, such that each row and column contains at least one non-zero element. The matrix $P$ is called a sandwich matrix. The multiplication rule in the Rees matrix semigroup $\mathcal{M}^{0}[G ; I, \Lambda ; P]=(I \times G \times \Lambda) \cup\{0\}$ is

$$
\begin{aligned}
(i, a, \lambda)(j, b, \mu) & = \begin{cases}\left(i, a p_{\lambda i} b, \mu\right) & \text { if } p_{\lambda i} \neq 0, \\
0 & \text { if } p_{\lambda i}=0,\end{cases} \\
(i, a, \lambda) 0 & =0(i, a, \lambda)=00=0 .
\end{aligned}
$$

A semigroup is called combinatorial if it contains no nontrivial subgroup. A completely 0 -simple semigroup is combinatorial if $G=\{1\}$. Here, 1 denotes the identity element of $G$. In the case of combinatorial completely 0 -simple semigroups for the triple $(i, 1, \lambda)$ we write $[i, \lambda]$. The two semigroups playing the most important roles among completely 0 -simple semigroups are the Brandt-semigroup $\mathbf{B}_{\mathbf{2}}=\mathcal{M}^{0}[G ;\{1,2\},\{1,2\} ; E]$, where $E$ is the $2 \times 2$ identity matrix and

$$
\mathbf{A}_{\mathbf{2}}=\mathcal{M}^{0}[G ;\{1,2\},\{1,2\} ; P], \text { where } P=\left(\begin{array}{ll}
1 & 1 \\
1 & 0
\end{array}\right) .
$$

Both semigroups are simple of type 5. The Brandt semigroup is both completely 0 simple and a so called 'inverse semigroup'. The authors proved in [6] that $\log \left|\mathbf{F}_{\mathcal{B}}(n)\right| \sim$ $2 n \log n$, where $\mathcal{B}$ denotes the variety generated by the Brandt semigroup. Let $\mathcal{A}$ denote the variety generated by $\mathbf{A}_{\mathbf{2}}$. Then $\mathcal{A}$ contains all combinatorial 0 -simple semigroups. So the variety generated by all combinatorial completely 0 -simple semigroups is generated by $\mathbf{A}_{2}$. In this paper we investigate the free spectra of $\mathcal{A}$. We prove that $\left|\mathbf{F}_{\mathcal{A}}(n)\right| \sim$ $2^{n^{2}+2 \log n}$. We do it via associating directed graphs to terms over $\mathbf{A}_{2}$. 


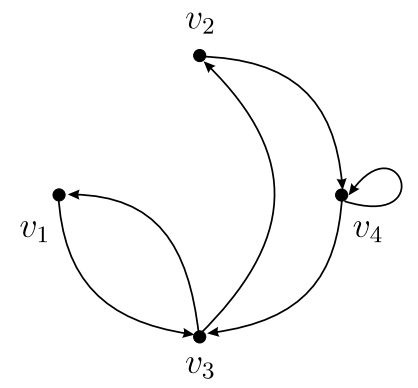

Figure 1. $t=x_{1} x_{3} x_{2} x_{4} x_{4} x_{3} x_{1} x_{3} x_{2}$.

2. Preliminaries. A term on an algebra $\mathbf{A}$ is an expression that can be obtained using (iterated) compositions of the basic operations and projections. The projections are the trivial operations $p_{i}^{n}\left(x_{1}, \ldots, x_{n}\right)=x_{i}$.

Two terms $t_{1}$ and $t_{2}$ are called equivalent $\left(t_{1}\left(x_{1}, \ldots, x_{n}\right) \equiv t_{2}\left(x_{1}, \ldots, x_{n}\right)\right.$ or shortly $t_{1} \equiv t_{2}$ ) over an algebra $\mathbf{A}$ if the term operations $t_{1}^{\mathbf{A}}$ and $t_{2}^{\mathbf{A}}$ are equal, i.e., for every $\bar{a} \in A^{n}, t_{1}^{\mathbf{A}}(\bar{a})=t_{2}^{\mathbf{A}}(\bar{a})$.

For an algebra $\mathbf{A}$ the set of $n$-ary term operations is $\mathrm{Clo}_{n} \mathbf{A}$. If $\mathcal{V}$ is the variety generated by $\mathbf{A}$ then $\left|\mathbf{F}_{\mathcal{V}}(n)\right|=\left|\mathrm{Clo}_{n} \mathbf{A}\right|$ for all $n$.

Let $t=t\left(x_{1}, \ldots, x_{n}\right)$ be an $n$-ary term. A term operation $t^{\mathbf{A}}$ is said to be essentially $n$-ary, if it depends on all of its variables, i.e. if for all $1 \leq i \leq n$ there exist $a_{1}, \ldots, a_{i-1}, a, b, a_{i+1}, \ldots, a_{n} \in A$ such that

$$
t\left(a_{1}, \ldots, a_{i-1}, a, a_{i+1}, \ldots, a_{n}\right) \neq t\left(a_{1}, \ldots, a_{i-1}, b, a_{i+1}, \ldots, a_{n}\right) .
$$

For $n \geq 1$, denote the set of essentially $n$-ary term operations over $\mathbf{A}$ by $E_{n}(\mathbf{A})$, while $E_{0}(\mathbf{A})$ denotes the set of all constant unary term operations of $\mathbf{A}$. Now we define $p_{n}(\mathbf{A})=\left|E_{n}(\mathbf{A})\right|$. Thus the equations $\left|\mathbf{F}_{\mathcal{V}}(n)\right|=\left|\mathrm{Clo}_{n} \mathbf{A}\right|=\sum_{k=0}^{n}\left(\begin{array}{l}n \\ k\end{array}\right) p_{k}(\mathbf{A})$ hold.

By the word graph we shall mean a directed graph without multiple edges. For a graph $G$ we shall denote the vertices and edges by $V(G)$ and $E(G)$, respectively. We say that a graph $G$ contains an Eulerian walk if there exists a directed walk in $G$ which contains all edges, possibly several times.

3. Term equivalence over $\mathbf{A}_{2}$. Recall that the sandwich matrix of the combinatorial 0-simple semigroup $\mathbf{A}_{\mathbf{2}}$ is

$$
P=\left(\begin{array}{ll}
1 & 1 \\
1 & 0
\end{array}\right),
$$

the index sets are $\Lambda=I=\{1,2\}$ and $[i, \lambda][j, \gamma]=0$ if and only if $\lambda=j=2$.

Let $t\left(x_{1}, x_{2}, \ldots, x_{k}\right)=t$ be a term over $\mathbf{A}_{2}$. Let $G(t)$ denote the following directed graph: $G(t)$ has $k$ many vertices $\left(v_{1}, v_{2}, \ldots, v_{k}\right)$. There is an edge between $v_{i}$ and $v_{j}$ if $x_{j}$ follows $x_{i}$ somewhere in $t$ that is, if $x_{i} x_{j}$ is a subword of $t$. For example, we get Figure 1 for the term $t=x_{1} x_{3} x_{2} x_{4} x_{4} x_{3} x_{1} x_{3} x_{2}$. Constructions of graphs related to semigroup terms can be found in [2], [5], [7], [10], [12],

Proposition 3.1 is formulated in [12], we omit its proof. 
Proposition 3.1. Let $t_{1}\left(x_{1}, x_{2}, \ldots, x_{k}\right)$ and $t_{2}\left(x_{1}, x_{2}, \ldots, x_{k}\right)$ be two terms over $\mathbf{A}_{2}$, such that $t_{1}=y_{1} y_{2} \ldots y_{m}$ and $t_{2}=z_{1} z_{2} \ldots z_{n}$, where $y_{i}, z_{j} \in\left\{x_{1}, x_{2}, \ldots, x_{k}\right\}$, for every $1 \leq i \leq m$ and $1 \leq j \leq n$. Then $t_{1} \equiv t_{2}$ if and only if the following hold:

(1) $G\left(t_{1}\right)=G\left(t_{2}\right)$;

(2) $y_{1}=z_{1}$;

(3) $y_{m}=z_{n}$.

4. The free spectrum of $\mathcal{A}$. The next lemma is consequence of Proposition 3.1.

LEMMA 4.1. Let $G$ be a directed graph with $n$ vertices and without multiple edges. If $G$ contains a closed Eulerian walk then there are exactly $n^{2}$ many non-equivalent essentially n-ary terms inducing the same graph.

Proof. Let $v_{1}, \ldots, v_{n}$ be the vertices of $G$. As there is a closed Eulerian walk in $G$, there is a closed Eulerian walk starting at $v_{j}: p_{1}=v_{j} \ldots v_{j}$ and a walk from $v_{j}$ to $v_{i}$ : $p_{2}=v_{j} \ldots v_{i}$. Then $p=x_{j} \ldots x_{i}$, the concatenation of the walks $p_{1}$ and $p_{2}$,

(1) covers all edges of $G$, as $p_{1}$ covers all edges of $G$;

(2) start by $v_{j}$;

(3) ends by $v_{i}$.

Thus the for corresponding term $t=x_{j} \ldots x_{i}$ we have

(1) $G(t)=G$;

(2) starts by $x_{j}$;

(3) ends by $x_{i}$.

We can repeat this construction for any $1 \leq i, j \leq n$, hence we have $n^{2}$ many distinct terms inducing $G$. On the other hand, by Proposition 3.1 we have listed all possible terms inducing $G$.

Proposition 4.2. Let $D(n)$ denote the number of directed graphs on $n$ vertices with a closed Eulerian walk. Then $D(n)=o\left(2^{n^{2}}\right)$.

Proof. Let $G$ be a digraph and let $|G|=n$. If $G$ does not contain a closed Eulerian walk, then there is a subset $S \subset V(G)$ such that there is no edge from $S$ to $V(G) \backslash S$. Let $S$ be a subset of size $k$. Then there are $2^{k^{2}}$ many graphs on $S, 2^{(n-k)^{2}}$ many graphs on $V(G) \backslash S$ and $2^{(n-k) k}$ possibilities for edges from $V(G) \backslash S$ to $S$. Hence there are $2^{k^{2}} 2^{(n-k)^{2}} 2^{(n-k) k}$ digraphs without outgoing edges from $S$.

For $k=1$ and $k=n-1$ we have

$$
\begin{aligned}
\left(\begin{array}{l}
n \\
1
\end{array}\right) 2^{1^{2}} 2^{(n-1)^{2}} 2^{(n-1) 1} & +\left(\begin{array}{c}
n \\
n-1
\end{array}\right) 2^{(n-1)^{2}} 2^{(1)^{2}} 2^{(n-1) 1} \\
& =2 \cdot n 2^{n^{2}-n+1} \leq n 2^{n^{2}-n+4} .
\end{aligned}
$$

Moreover $\left(\begin{array}{l}n \\ k\end{array}\right) \leq 2^{n}$ implies

$$
\left(\begin{array}{l}
n \\
k
\end{array}\right) 2^{k^{2}} 2^{(n-k)^{2}} 2^{(n-k) k} \leq 2^{n} 2^{k^{2}} 2^{(n-k)^{2}} 2^{(n-k) k}=2^{n^{2}+n-n k+k^{2}} .
$$

In case $1<k<n-1$

$$
2^{n^{2}+n-n k+k^{2}} \leq 2^{n^{2}-n+4}
$$


Let $N(n)$ denote the number of directed graphs without a closed Eulerian walk on $n$ vertices. Then

$$
N(n) \leq \sum_{1}^{n-1}\left(\begin{array}{l}
n \\
k
\end{array}\right) 2^{k^{2}} 2^{(n-k)^{2}} 2^{(n-k) k} .
$$

Using the above estimates we obtain the following upper bound for $N(n)$ :

$$
N(n) \leq n 2^{n^{2}-n+4}+(n-2) 2^{n^{2}-n+4} \leq(2 n-2) 2^{n^{2}-n+4} .
$$

As $N(n)+D(n)=2^{n^{2}}$, we have $D(n)=o\left(2^{n^{2}}\right)$.

THEOREM 4.3 .

$$
\left|\mathbf{F}_{\mathcal{A}}(n)\right|=o\left(2^{n^{2}+2 \log n}\right) .
$$

Proof. By Proposition 3.1 terms correspond to directed graphs and pairs of vertices, hence $\left|\mathbf{F}_{\mathcal{A}}(n)\right| \leq n^{2} 2^{n^{2}}$ holds. From Lemma 4.1 and from Proposition 4.2 we get $p_{n}\left(\mathbf{A}_{2}\right)=n^{2} \cdot o\left(2^{n^{2}}\right)$ for the number of the essentially $n$-ary terms. Since $p_{n}\left(\mathbf{A}_{2}\right) \leq\left|\mathbf{F}_{\mathcal{A}}(n)\right|$ we have:

$$
\left|\mathbf{F}_{\mathcal{A}}(n)\right|=o\left(2^{n^{2}+2 \log n}\right)
$$

There is still plenty of work to be done. We gave an asymptotic value for the free spectrum of the variety generated by the combinatorial 0 -simple semigroups. One should ask for the free spectra of a variety generated by a (not necessarily combinatorial) 0 -simple semigroup in general. It seems that even a very special case looks to be too complicated.

Problem Let $G$ be an arbitrary (abelian, nilpotent) group. Find the free spectra of $\mathcal{M}^{0}[G ; I, \Lambda ; P]$ where $P$ is a $0-1$ matrix.

Acknowledgements The research of the authors was supported by the Hungarian National Foundation for Scientific Research, Grants NK67867 and NK67870.

\section{REFERENCES}

1. J. Berman, Free spectra gaps and tame congruence, Int. J. Algebra Comput. 5 (1995), no. $6,651-672$.

2. L. R. Graham, On finite 0 -simple semigroups and graph theory, Math. Syst. Theory 2 , (1968), 325-339.

3. G. Higman, The order of relatively free groups, in Proceedings of the International Conference on the Theory of Groups, Canberra 1965 (Gordon and Breach, 1967), 153-163.

4. D. Hobby and R. McKenzie, The structure of finite algebras, Contemporary Mathematics No. 76 (Amer. Math. Soc., Providence, 1988).

5. C. H. Houghton, Completely 0 -simple semigroups and their associated graphs and groups, Semigroup Forum 37 (1977), 41-67.

6. K. Kátai-Urbán, and Cs. Szabó, Free spectrum of the variety generated by the five element combinatorial Brandt semigroup, Semigroup Forum, to appear 
7. G. Mashevitzky, Completely simple and completely 0-simple semigroup identities, Semigroup Forum 37 (1988), 253-264.

8. P. Neumann, Some indecomposable varieties of groups, Quart. J, Math., Oxford Ser. 2 14 (1963), 46-50.

9. M. Petrich, Inverse semigroups (Wiley and Sons, New York, 1984).

10. S. Seif and Cs. Szabó, Algebra complexity problems involving graph homomorphisms, semigroups and the constraint satisfaction problem, J. Complexity 19 (2003), 153-160.

11. S. Seif, and Cs. Szabó, Computational complexity of checking identities in 0 -simple semigroups and matrix semigroups over finite fields, Semigroup Forum (to appear).

12. A. N. Trahtman, Identities of a five-element 0-simple semigroup, Semigroup Forum $\mathbf{4 8}$ (1994), 385-387. 